[RADIOCARBon, Vol 22, No. 1, 1980, P 99-107]

\title{
UNIVERSITY OF MIAMI RADIOCARBON DATES XVII
}

\section{R JOHNSON, D S INTRONE, J MATTES, and J J STIPP \\ Department of Geology, University of Miami \\ Coral Gables, Florida 33124}

The following radiocarbon dates are a partial list of samples measured for a variety of projects and materials since April 1979. Chemical and counting procedures remain the same as indicated in $\mathrm{R}, \mathrm{v} 20, \mathrm{p} 274-$ 282.

Calculations are based on the 5568 year Libby ${ }^{14} \mathrm{C}$ half-life. Precision is reported as one-standard deviation based only on statistical counting uncertainties in the measurement of the background, NBS modern standard, and sample activities. $\delta^{13} \mathrm{C}$ values are measured relative to PDB and reported ages are corrected for isotopic fractionation by normalizing to $-25 \%$. An additional 400 year reservoir age correction has been applied to marine carbonates.

\section{SAMPLE DESCRIPTIONS}

\section{GEOLOGIC SAMPLES}

\section{Virgin Island Series}

Core samples of carbonate sand and coral rock taken from $S$ shelf of US Virgin Islands were dated to determine sedimentation rates. Coll 1978 and subm 1979 by R Miller, USGS, Corpus Christi, Texas.

UM-1728. 305020

$1750 \pm 90$

Carbonate sand $\left(18^{\circ} 19^{\prime} \mathrm{N}, 65^{\circ} 1.25^{\prime} \mathrm{W}\right)$ coll by $\mathrm{R}$ Miller.

UM-1729. 380095

$$
2020 \pm 80
$$

Carbonate sand $\left(18^{\circ} 19^{\prime} \mathrm{N}, 65^{\circ} 1.25^{\prime} \mathrm{W}\right)$ coll by $\mathrm{R}$ Miller.

UM-1730. 3155170

$2890 \pm 90$

Carbonate sand $\left(18^{\circ} 19^{\prime} \mathrm{N}, 65^{\circ} 1.25^{\prime} \mathrm{W}\right)$ coll by $\mathrm{R}$ Miller.

UM-1731. 3230245

$3660 \pm 80$

Carbonate sand $\left(18^{\circ} 19^{\prime} \mathrm{N}, 65^{\circ} 1.25^{\prime} \mathrm{W}\right)$ coll by R Miller.

UM-1732. 3320335

$5940 \pm 85$

Carbonate sand $\left(18^{\circ} 19^{\prime} \mathrm{N}, 65^{\circ} 1.25^{\prime} \mathrm{W}\right)$ coll by $\mathrm{R}$ Miller.

UM-1733. H-861-I

$\mathbf{2 2 , 3 7 0} \pm \mathbf{2 8 0}$

Carbonate sand $\left(18^{\circ} 20.5^{\prime} \mathrm{N}, 64^{\circ} 58^{\prime} \mathrm{W}\right)$ coll by Woodward and Clyde.

UM-1734. Rotary Core \#4

$7600 \pm 100$

Coral rock $\left(18^{\circ} 12^{\prime} \mathrm{N}, 64^{\circ} 44^{\prime} \mathrm{W}\right)$ coll by $\mathrm{R}$ Miller.

\section{Carteret County series}

Core samples of organic clay and shell taken from a lagoonal and back barrier environment in Carteret Co, North Carolina. Samples coll 
from Back Sound and Harkers I. (34 $40^{\prime} \mathrm{N}, 76^{\circ} 40^{\prime} \mathrm{W}$ ) for stratigraphic correlation. Coll July 1978 by W Berelson; subm by D Heron, Duke Univ, Durham, North Carolina.

UM-1647. H I \#11 188 to $191 \mathrm{~cm}$ $4300 \pm 90$

Oyster shell within lagoonal mud facies (Back Sound).

UM-1648. H I \#26 $213 \mathrm{~cm}$

$3080 \pm 90$ Sound).

Gastropod shells from marsh mud below tidal delta deposit (Back

UM-1649. H I \#37 208 to $218 \mathrm{~cm}$

$1520 \pm 70$

Clam shells near top of marsh mud below tidal delta deposit in back barrier environment (Back Sound).

UM-1650. H I \#18 257 to $262 \mathrm{~cm}$

$15,440 \pm 280$ ment.

Organic clay unit with lagoonal mud facies, back barrier environ-

UM-1651. H I 25b, 475 to $483 \mathrm{~cm}$

$>32,000$

Organic stained sandy clay near top of lagoonal mud, back barrier environment.

UM-1652. H I A 457 to $483 \mathrm{~cm}$ I.).

Humate from soil zone below modern barrier island sands (Harkers

\section{Back Sound series}

Shell and organic sediment coll via cores from marsh, marine, and back barrier island environments in Back Sound, North Carolina $\left(36^{\circ}\right.$ $\left.40^{\prime} \mathrm{N}, 76^{\circ} 30^{\prime} \mathrm{W}\right)$. Samples dated to determine stratigraphic correlation. Coll 1978 by W Berelson and subm 1979 by D Heron, Duke Univ, Durham, North Carolina. UM-1719 coll and subm 1979 by W Berelson.

UM-1707. F (335 to $427 \mathrm{~cm})$

32,540 $+680$

Mercenaria sp fragments from marsh mud.

$$
-630
$$

UM-1708. 12 (112 to $142 \mathrm{~cm})$

$1050 \pm 140$

Oyster and slipper shells from marsh mud.

UM-1709. 24 (287 to $295 \mathrm{~cm})$

$3190 \pm 150$

Oyster and slipper shells from silty mud in marsh environment.

UM-1710. 5 (76cm)

$2940 \pm 150$

Gastropod shells from coarse lag in mixed marine environment.

UM-1711. 16 (10 to $20 \mathrm{~cm}$ )

$2720 \pm 135$ ment.

Ribbed clam shell from coarse lag inlet fill in mixed marine environ- 
UM-1712. 29 (Bottom 91cm)

$3580 \pm 110$

Ribbed clam shells from coarse lag inlet fill in mixed marine-backbarrier environment.

UM-1713. 23 ( 183 to $188 \mathrm{~cm}$ )

$2710 \pm 150$

Peaty clay from buried peaty marsh mud unit.

UM-1714. 19 (76cm)

$3780 \pm 140$

Mix of shell fragments in buried marsh mud.

UM-1715. 13 (0 to $10 \mathrm{~cm})$

$12,550 \pm 300$

Organic clay from buried marsh mud.

UM-1716. 13 (241 to $246 \mathrm{~cm})$

$+380$

9090

$-360$

Organic sand from humic zone in barrier island sand.

UM-1919. 39 ( 56 to $64 \mathrm{~cm}$ )

$4800 \pm 100$

Oyster, razor and Mercenaria sp clam shells buried in muddy marsh unit below fine sandy horizon.

\section{Harkers Island series}

Shell samples coll from mud-marsh unit on Harkers I., North Carolina $\left(34^{\circ} 40^{\prime} \mathrm{N}, 76^{\circ} 30^{\prime} \mathrm{W}\right)$. Samples dated to determine stratigraphic correlation in area. Coll 1978 and subm 1979 by W Berelson, Duke Univ, Durham, North Garolina.

UM-1717. I ( 457 to $518 \mathrm{~cm})$

$$
28,940 \begin{array}{r}
+500 \\
-470
\end{array}
$$

Oyster shell from marsh unit below barrier island sands.

UM-1718. J (1524 to $1585 \mathrm{~cm})$ $+625$ 23,060 $-\mathbf{5 8 0}$

Gemma gemma from marsh unit below barrier island sands.

\section{Lake Erie series}

Wood samples taken from cores in Lake Erie NW of Erie, Pennsylvania. Samples dated to determine age of ridge offshore Presque Isle. Coll and subm 1979 by S Williams, Coastal Engineering Research Center, Fort Belvoir, Virginia.

UM-1703. Core $4-104 \mathrm{~cm}$

$$
\begin{array}{r}
\mathbf{8 2 4 0} \pm \mathbf{2 1 0} \\
\delta^{13} C=-25.06 \%
\end{array}
$$
$\left.18^{\prime} \mathrm{W}\right)$.

Sample from silty sand matrix at water depth, $20.4 \mathrm{~m}\left(42^{\circ} 15^{\prime} \mathrm{N}, 80^{\circ}\right.$

UM-1704. Core $18-357 \mathrm{~cm}$

$$
\begin{array}{r}
6870 \pm 150 \\
\delta^{1 s} C=-29.45 \% \text { o }
\end{array}
$$

Sample from fine to medium sand matrix at water depth, $18.5 \mathrm{~m}$ $\left(42^{\circ} 12^{\prime} \mathrm{N}, 80^{\circ} 17^{\prime} \mathrm{W}\right)$. 
UM-1705. Core $23-219 \mathrm{~cm}$

Sample from matrix at water depth, $19.2 \mathrm{~m}\left(42^{\circ} 12^{\prime} \mathrm{N}, 80^{\circ} 18^{\prime} \mathrm{W}\right)$.

UM-1706. Core $28-351 \mathrm{~cm} \quad 10,800 \pm 190$ $\delta^{13} \mathrm{C}=-26.20 \%$ o $\left.09^{\prime} \mathrm{W}\right)$.

Sample from silt-clay matrix at water depth, $18.4 \mathrm{~m}\left(42^{\circ} 12^{\prime} \mathrm{N}, 80^{\circ}\right.$

\section{Point Delgada series}

Wood and charcoal samples from fluvial siltstones above marine platform out on cretaceous bedrock at Point Delgada, California $\left(40^{\circ} 1^{\prime} \mathrm{N}\right.$, $\left.124^{\circ} 4^{\prime} \mathrm{W}\right)$. Dates uplift and sedimentation rates for fluvial sediments above the platform and may record rise in sea level, ante-dating elev of terrace. Coll and subm 1979 by R McLaughlin, USGS, Menlo Park, California.

UM-1631. SK 79-1

$$
\begin{aligned}
& +730 \\
& \text { 34,380 } \\
& +610 \\
& \text { 29,040 } \\
& \delta^{13} \mathrm{C}=-24.48 \% \text { 。 }
\end{aligned}
$$

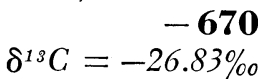

Wood.

UM-1632. SK 79-2

Charcoal from same location as UM-1631.

\section{Alaska Foodweb series}

Samples of various living organisms were dated to establish a modern marine standard for pelagic arctic fish and marine plants and an estimation of carbon input resulting from detritus based foodweb utilizing eroded peat. Subm 1979 by D Schell, Univ Alaska, Fairbanks, Alaska.

UM-1738. 79.7

$105.0 \%$ modern $\delta^{13} \mathrm{C}=-17.47 \%$

Laminaria stipes and holdfast coll by D Schell in Stefannsson Sound $\left(70^{\circ} 23^{\prime \prime} \mathrm{N}, 147^{\circ} 40^{\prime \prime} \mathrm{W}\right)$.

UM-1739. 79.8

107.1\% modern ${ }^{13} \mathrm{C}=-15.04 \%$

Laminaria blades coll by D Schell in Stefannsson Sound $\left(70^{\circ} 23^{\prime \prime} \mathrm{N}\right.$, $147^{\circ} 40^{\prime \prime} \mathrm{W}$ ).

UM-1740. 79.9

104.4\% modern $\delta^{13} C=-20.82 \%$ o

Myoxocephalus quadricornus muscle tissue coll by J Helmericks in Harrison Bay $\left(70^{\circ} 30^{\prime \prime} \mathrm{N}, 150^{\circ} 10^{\prime \prime} \mathrm{W}\right)$. 
UM-1741. 79-10

103.7\% modern $\delta^{13} \mathrm{C}=-21.60 \%$

Arctic cod muscle tissue coll by LGL Ltd in Steffannsson Sound $\left(70^{\circ} 23^{\prime \prime} \mathrm{N}, 147^{\circ} 40^{\prime \prime} \mathrm{W}\right)$.

\section{Puget Sound series}

Peat and wood fragments hand picked from unconsolidated sediments exposed in marine coastal bluffs on Whidbey I., Washington. Dated to provide temporal framework for stratigraphy of area. Coll and subm 1979 by K L Stoffel, Dept Nat Resources, Olympia, Washington.

$$
\begin{aligned}
& \begin{array}{lllll}
\text { UM-1745. } & \text { 29NO3E } & 29.8 \mathrm{~g} & \text { UBE\# } 1 & 28,910 \\
& & 630
\end{array} \\
& +690 \\
& \delta^{13} C=-27.38 \% \\
& \text { UM-1746. } 29 \text { NO3E } \quad 29.8 \mathrm{~g} \quad \text { UBE\#2 } 29,330 \\
& +550 \\
& \text { UM-1746. } \mathbf{5 2 0} \\
& \delta^{13} C=-26.72 \% \\
& +1730 \\
& \delta^{13} \mathrm{C}=-\mathbf{- 1 4 2 0}
\end{aligned}
$$

Peat samples UM-1745-1747 from Useless Bay East $\left(47^{\circ} 58^{\prime} 12^{\prime \prime} \mathrm{N}\right.$, $122^{\circ} 27^{\prime} 36^{\prime \prime}$ S) dated to determine age of Whidbey formation.

UM-1748. 28NO3E $\quad 16.4$ SH\#I $\quad 29,130$

$$
+570
$$

$\delta^{1 s} C=$

Peat from Scatchet Head ( $47^{\circ} 55^{\prime} 03^{\prime \prime}$ N, $122^{\circ} 25^{\prime} 47^{\prime \prime}$ S).

UM-1749. 28N03E 16.5h SH\#2 241,500

Peat from Scatchet Head ( $\left.47^{\circ} 55^{\prime} 14^{\prime \prime} \mathrm{N}, 122^{\circ} 25^{\prime} 50^{\prime \prime} \mathrm{N}\right)$.

UM-1750. 28N03E 16.4h SH\#3 $\quad>39,600$

Wood fragments from Scatchet Head $\left(47^{\circ} 55^{\prime} 11^{\prime \prime} \mathrm{N}, 122^{\circ} 25^{\prime} 42^{\prime \prime} \mathrm{N}\right)$.

UM-1751. 28N03E 23.3h PP\# $1 \quad 30,470$

$$
+1650
$$

$-1370$

$\delta^{13} \mathrm{C}=-28.68 \%$ o $\left.56^{\prime \prime} \mathrm{S}\right)$.

Peat taken from base of Possession Drift $\left(47^{\circ} 53^{\prime} 34^{\prime \prime} \mathrm{N}, 122^{\circ} 22^{\prime}\right.$

UM-1752. 29N02E 26.5f DBE\# 1

$$
\begin{array}{r}
\mathbf{2 3 , 6 0 0} \pm \mathbf{2 8 0} \\
\delta^{13} C=-26.97 \%
\end{array}
$$

Wood fragments from Whidbey Esperance contact $\left(47^{\circ} 58^{\prime} 29^{\prime \prime} \mathrm{N}\right.$, $\left.122^{\circ} 31^{\prime} 08^{\prime \prime} \mathrm{W}\right)$. 
UM-1753. 29N02E 27.3c DBW\#1

Peat from S Whidbey I. $\left(47^{\circ} 58^{\prime} 12^{\prime \prime} \mathrm{N}, 122^{\circ} 32^{\prime} 07^{\prime \prime} \mathrm{W}\right)$.

$\delta^{13} \mathrm{C}=-\mathbf{- 2 8 2 8 0}$

UM-1754. 32NO1E 07.6d WI\#I

Wood fragment coll by G W Thorsen from NW Whidbey I. $\left(48^{\circ}\right.$ $\left.16^{\prime} 29^{\prime \prime} \mathrm{N}, 122^{\circ} 44^{\prime} 15^{\prime \prime} \mathrm{W}\right)$. Dated to determine min age of Vashon continental ice at this lot.

UM-1692. GN-1

$$
\delta^{1 s} C=-26.30 \% \text { o }
$$

Wood sample from $3 \mathrm{~m}$ below surface in Williston Quad, Florida $\left(29^{\circ} 27^{\prime} 0^{\prime \prime} \mathrm{N}, 82^{\circ} 23^{\prime} 0^{\prime \prime} \mathrm{W}\right)$ was dated to study preservation of organic material in peat. Coll 1977 by G Neal; subm 1979 by B Purdy, Florida State Mus, Univ Florida, Gainesville.

UM-1693. V26-176, 550-570cm

$$
24,750 \begin{aligned}
& +1560 \\
& -1300
\end{aligned}
$$

Calcareous lutite cored from lower Continental Rise $\left(36^{\circ} 03^{\prime} \mathrm{N}, 72^{\circ}\right.$ $23^{\prime} \mathrm{W}$ ) was dated to study frequency spectrum analysis of faunal fluctuation. Coll by Lamont-Doherty Geol Observatory; subm 1979 by D Schnitker, Dept Oceanog, Univ Maine, Walpole.

$$
\text { II. ARCHAEOLOGIC SAMPLES }
$$

\section{Columbia County series}

Charred wood samples coll from Indian mound in Columbia Co, Florida. Samples date construction and charnel activities assoc with Mound A at 8-Co-17 (McKeithen) a Weeden Island site. Sample coll Feb 1979 and subm April 1979 by J Milanich, Florida State Mus, Gainesville.

UM-1642. 8 - Co - 17 FS 1147

$1770 \pm 70$

Charred sample (Prunus sp) from post in burial preparation area, top of primary mound.

UM-1643. 8 - Co - 17 FS 1191

$$
1450 \pm 90
$$

Charred sample (Pinus sp) coll from post lying horizontally beside primary mound.

UM-1644. 8 - Co - 17 FS 1267

$1690 \pm 90$ mound.

Sample from small oval hearth used during construction of primary

UM-1645. 8 - Co - 17 FS

$1530 \pm 80$

Sample from fire pit that was intrusive to primary mound. 
UM-1646. 8 - Co - 17 FS 1312

$1520 \pm 70$

Sample (Pinus sp) from post erected in sloppng trench intrusive to primary mound.

\section{Lumpkin County Pottery series}

Charred wood coll from exposed surface during excavation in Lumpkin Co, Georgia ( $\left.34^{\circ} 28^{\prime} 07^{\prime \prime} \mathrm{N}, 83^{\circ} 58^{\prime} 14^{\prime \prime} \mathrm{W}\right)$. Samples dated to obtain absolute ages for different pottery phases. Coll and subm 1979 by M Crook, West Georgia Coll, Carrollton.

UM-1675. FEATURE 14; \#267/1

$$
\begin{array}{r}
980 \pm 100 \\
\delta^{13} C=-27.00 \% \text { o }
\end{array}
$$

Wood sample from hearth assoc with Woodstock Complicated Stamped pottery. Indicates Early Mississippian period.

UM-1676. FEATURE $10 ; \# 102 / 1$

$$
\delta^{13} C \begin{array}{r}
\mathbf{2 4 3 0} \pm \mathbf{7 0} \\
=-27.22 \% \text { o }
\end{array}
$$

Charred wood assoc with stone-lined earth oven and Cartersville Check-Stamped pottery. Earth oven is considered to date to Woodland period.

UM-1677. FEATURE 15; \#372/1

$$
\begin{array}{r}
1420 \pm 80 \\
\delta^{13} C=-26.24 \% o
\end{array}
$$

Wood assoc with Mossey-Oak Simple Stamped, Cartersville CheckStamped, and Swift Creek Complicated Stamp types. Sample from refuse pit which appears to date to Woodland period.

\section{Granada series}

Charcoal samples from Granada site (8 Da 11) Dade Co, Miami, Florida $\left(80^{\circ} 11^{\prime} 25^{\prime \prime} \mathrm{W}, 25^{\circ} 46^{\prime} 21^{\prime \prime} \mathrm{N}\right)$. Dated to revise ceramic chronology of SE Florida and to date assoc artifacts. Coll and subm 1979 by $\mathrm{S}$ Bonath, Bur Hist Sites \& Properties, Tallahassee, Florida.

UM-1660. C-79-1

$$
1080 \pm 50
$$

+2.08 to $1.69 \mathrm{~m}$ in $6 \mathrm{~S}-12 \mathrm{~W}$.

UM-1661. C-79-2

$$
530 \pm 60
$$

+2.10 to $1.95 \mathrm{~m}$ in $2 \mathrm{~S}-16 \mathrm{~W}$.

UM-1662. C-79-3

$1020 \pm 70$

+1.70 to $1.55 \mathrm{~m}$ in $10 \mathrm{~S}-16 \mathrm{~W}$.

UM-1663. C-79-4

$1160 \pm 50$

+1.55 to $1.40 \mathrm{~m}$ in $10 \mathrm{~S}-16 \mathrm{~W}$.

UM-1664. C-79-S

$1030 \pm 50$

+1.40 to $1.18 \mathrm{~m}$ in $10 \mathrm{~S}-16 \mathrm{~W}$.

UM-1665. C-79-6

$1350 \pm 80$

+1.30 to $1.15 \mathrm{~m}$ in $10 \mathrm{~S}-16 \mathrm{~W}$. 
UM-1666. C-79-7

$1090 \pm 70$

+1.80 to $1.47 \mathrm{~m}$ in $0 \mathrm{~N}-72 \mathrm{~W}$.

UM-1667. C-79-8

$490 \pm 110$

+1.70 to $1.27 \mathrm{~m}$ in $0 \mathrm{~N}-72 \mathrm{~W}$.

UM-1668. C-79-9

$890 \pm 60$

+1.86 to $1.51 \mathrm{~m}$ in $0 \mathrm{~N}-70 \mathrm{~W}$.

UM-1669. C-79-10

$1230 \pm 80$

+1.93 to $1.56 \mathrm{~m}$ in $0 \mathrm{~N}-70 \mathrm{~W}$.

UM-1670. C-79-11

$1020 \pm 50$

+1.75 to $1.60 \mathrm{~m}$ in $2 \mathrm{~S}-64 \mathrm{~W}$.

UM-1671. C-79-12

+1.58 to $1.15 \mathrm{~m}$ in $0 \mathrm{~N}-74 \mathrm{~W}$.

$1220 \pm 50$

UM-1672. C-79-13

$1930 \pm 75$

+1.55 to $1.40 \mathrm{~m}$ in $2 \mathrm{~S}-64 \mathrm{~W}$.

UM-1674. C-79-15

+2.06 to $1.85 \mathrm{~m}$ in $2 \mathrm{~S}-10 \mathrm{~W}$.

$$
1440 \pm 140
$$

\section{UM-1742. AE 35}

$940 \pm 60$

Busycon contratium shell artifact recovered from Aqui Esta burial mound on north fork of Alligator Creek, $2 \mathrm{~km} \mathrm{~S}$ of Punta Gorda, Florida $\left(26^{\circ} 53^{\prime} 52^{\prime \prime} \mathrm{N}, 82^{\circ} 02^{\prime} 40^{\prime \prime} \mathrm{W}\right)$. Dated to determine time of use and correlation with Safety Harbor culture. Coll 1962 by R Jones; subm 1979 by G Luer, Sarasota, Florida.

\section{Dade County series}

Charcoal, shell, and peat from various archaeol sites in Dade Co, Florida were dated to determine sequence of Indian habitation. Coll by R Carr, I Eyster, W Carson and J Beriault; subm 1979 by Dade Co Hist Survey.

\section{UM-1720. Trail site-marine shell}

$$
\begin{array}{r}
\mathbf{2 4 4 0} \pm \mathbf{7 0} \\
\delta^{13} C=+1.21 \% \text { o }
\end{array}
$$

Strombus gigas coll from Trail Site, 8 Da34 $\left(25^{\circ} 45^{\prime} \mathrm{N}, 80^{\circ} 23^{\prime} 27^{\prime \prime}\right.$ $\mathrm{W})$ to date occupation on lowest level of Everglades midden.

UM-1721. FS \#218

$$
\begin{array}{r}
\mathbf{2 0 0 0} \pm \mathbf{7 0} \\
\delta^{13} C=-27.25 \%
\end{array}
$$

Charcoal from Pig site, 8Da1057, Test Pit \#1, LVL8 (25 49' 14" N, $\left.80^{\circ} 23^{\prime} 03^{\prime \prime} \mathrm{W}\right)$ to date Indian habitation of Everglades tree island. 
UM-1722. Pig site-fresh water shell

Shell coll at Pig site, 8Da1057, Test Pit \#1, 90 to $93 \mathrm{~cm}\left(25^{\circ} 49^{\prime} 14^{\prime \prime}\right.$ $\left.\mathrm{N}, 80^{\circ} 23^{\prime} 27^{\prime \prime} \mathrm{W}\right)$ to understand forrnation of tree island.

\section{UM-1723. Pig site-peat}

$\mathbf{3 7 7 0} \pm \mathbf{8 0}$

$\delta^{13} C=-24.92 \%$

Peat taken from Pig site, 8 Dal057, Test Pit \#1, 90 to $100 \mathrm{~cm}\left(25^{\circ}\right.$ $49^{\prime} 14^{\prime \prime} \mathrm{N}, 80^{\circ} 23^{\prime} 27^{\prime \prime} \mathrm{W}$ ) to date pre-occupation period of Everglades tree island.

UM-1724. FS \#234

$$
\begin{gathered}
1460 \\
-180 \\
\delta^{13} C=-26.49 \%
\end{gathered}
$$

Charcoal recovered from Beal Smith site, 8Da1043, Test Pit \#1, Level 3.

UM-1725. FS \#235

$$
\begin{aligned}
& +120 \\
& 1890 \\
& \delta^{13} C=-25.87 \% \text { o }
\end{aligned}
$$

Charcoal coll from Beal Smith site, 8Da1043, Test Pit \#1, level 4, 30 to $40 \mathrm{~cm},\left(25^{\circ} 55^{\prime} 25^{\prime \prime} \mathrm{N}, 80^{\circ} 28^{\prime} 38^{\prime \prime} \mathrm{W}\right)$ to date occupational chronology on Everglades tree island.

UM-1726. FS \#242

$$
\begin{array}{r}
\mathbf{3 9 5 0} \pm \mathbf{9 0} \\
\delta^{13} C=+1.43 \%
\end{array}
$$

Busycon shell taken from Beal Smith site, 8Da1043, Test Pit \#1, Level 11,100 to $110 \mathrm{~cm},\left(25^{\circ} 55^{\prime} 25^{\prime \prime} \mathrm{N}, 80^{\circ} 28^{\prime} 38^{\prime \prime} \mathrm{W}\right)$ was dated to establish occupational sequence on Everglades tree island.

$$
\text { UM-1727. FS \#313 }
$$

$$
\delta^{13} C=-24.38 \%
$$

Charcoal recovered from Hog site, 8Da1045, Test Pit \#1, Level 8, 70 to $80 \mathrm{~cm},\left(25^{\circ} 53^{\prime} 47^{\prime \prime} \mathrm{N}, 80^{\circ} 32^{\prime} 37^{\prime \prime} \mathrm{W}\right)$ was dated for chronology of occupation.

REFEFENCE

Calvert, M, Rudolph, Kim, and Stipp, J J, 1978, University of Miami radiocarbon dates XII: Radiocarbon, v 20, p 274-282. 